\title{
Estudo Psicométrico da Versão Portuguesa do Questionário de Padrões de Comunicação - Versão Curta numa Amostra de Mulheres Heterossexuais
}

\author{
Psychometric Study of the Portuguese Version of the Communication Patterns \\ Questionnaire - Short Form in a Sample of Heterosexual Women
}

Tatiana Carvalhal ${ }^{1}$ e Bárbara Nazaré ${ }^{2}$

\begin{abstract}
Resumo
As interações conjugais caracterizam-se por dois tipos de padrões comunicacionais: complementares (e.g., exigir/afastar-se) e simétricos (e.g., comunicação construtiva mútua, evitamento mútuo). O Questionário de Padrões de Comunicação - Versão Curta (QPC-VC), de Christensen e Heavey, avalia padrões de comunicação conjugais. O presente estudo, de natureza transversal e observacional, explorou a estrutura fatorial e as características psicométricas da versão portuguesa do QPC-VC numa amostra de 164 mulheres heterossexuais, que preencheram os questionários online. Foi identificada uma estrutura fatorial diferente da original, composta por três subescalas: Padrão Simétrico Positivo, Padrão Alternado Negativo e Padrão Complementar Negativo. O QPC-VC mostrou boa validade fatorial, convergente e divergente e boa consistência interna. Os padrões comunicacionais negativos associaram-se a dificuldades conjugais, enquanto o padrão simétrico positivo constituiu um recurso conjugal. Assim, as intervenções conjugais devem promover o uso de estratégias de comunicação adaptativas (e.g., negociação), minimizando as estratégias potencialmente prejudiciais para a relação (e.g., acusações).
\end{abstract}

Palavras-chave: comunicação, conjugalidade, estudos psicométricos, exigir/afastar-se, Questionário de Padrões de Comunicação

\begin{abstract}
Couple interactions are characterized by two types of communication patterns: complementary (e.g., demand/withdraw) and symmetrical (e.g., mutual constructive communication, mutual avoidance). Christensen and Heavey's Communication Patterns Questionnaire - Short Form (CPQ-SF) aims to assess communication patterns in couples. This was a cross-sectional, observational study with the aim of exploring the factor structure and psychometric characteristics of the Portuguese version of the CPQ-SF. A sample of 164 heterosexual women answered the questionnaires online. The factor structure was different from the original and was composed of three subscales: Positive Symmetrical Pattern, Negative Alternate Pattern, and Negative Complementary Pattern. The questionnaire showed good factorial, convergent, and divergent validity, and good internal consistency. Negative communication patterns were associated with marital difficulties, while the positive symmetrical pattern was a resource for couples. Therefore, couple interventions should promote the use of adaptive communication strategies (e.g., negotiation), and minimize the use of potentially problematic strategies (e.g., blame).
\end{abstract}

Keywords: communication, Communication Patterns Questionnaire, couplehood, demand/withdraw, psychometric studies

\footnotetext{
${ }^{1}$ Mestre em Psicologia Clínica e da Saúde. Psicóloga Clínica na Clivip, Policlínica Médica. Rua Tierno Galvan, Torre 3, Piso 6, Sala 606, 1250-096, Lisboa, Portugal. Tel.: 910014569. E-mail: tatianacarvalhalpsi@ gmail.com

${ }^{2}$ Doutora em Psicologia da Saúde. Professora Auxiliar na Escola de Psicologia e Ciências da Vida da Universidade Lusófona de Humanidades e Tecnologias. Campo Grande, 376, 1749-024 Lisboa, Portugal. Tel.: 964126808. E-mail: barbara.nazare@ulusofona.pt

Revista Iberoamericana de Diagnóstico y Evaluación - e Avaliação Psicológica. RIDEP · №58 · Vol.1 · 169-182 - 2021

ISSN: 1135-3848 print /2183-6051online
} 


\section{Introdução}

A comunicação corresponde ao processo através do qual os elementos de um casal trocam informação, por via verbal (e.g., em conversas presenciais), não-verbal (e.g., através de expressões faciais) e/ou digital (e.g., por e-mail; Khaleque, 2018). A comunicação é central na formação e manutenção das relações (Meraz \& Palencia, 2012), pelo que uma boa comunicação é considerada pelos adultos como uma das principais razões para manter a relação conjugal (González, Aragón, Díaz-Loving, \& Ramírez, 2012). De forma congruente, problemas de comunicação e conflitos frequentes constituem das principais razões para os elementos do casal, principalmente as mulheres (Doss, Simpson, \& Christensen, 2004), procurarem terapia conjugal (Neumann \& Wagner, 2015; Roddy, Rothman, Cicila, \& Doss, 2019). Embora os problemas comunicacionais sejam tradicionalmente vistos como preditores das dificuldades conjugais, existe evidência empírica de que estas mesmas dificuldades podem potenciar o uso de estratégias de comunicação negativas (Nguyen, Williamson, \& Bradbury, 2017), ilustrando uma relação bidirecional entre as variáveis (Eldridge, Cencirulo, \& Edwards, 2017).

As interações que os elementos do casal estabelecem tendem a ser estáveis, consistindo em sequências de comunicação que, dada a sua repetição, são designadas como padrões (Sullaway \& Christensen, 1983). Na maioria dos estudos sobre dinâmicas familiares e conjugais, os padrões comunicacionais são concetualizados como simétricos ou complementares (Schrodt, Witt, \& Shimkowski, 2014), sendo variáveis no que toca à sua funcionalidade (Sullaway \& Christensen, 1983).

\section{Padrões de Comunicação Simétricos}

A simetria caracteriza as trocas entre parceiros com o mesmo nível de poder (Watzlawick, 2014) e traduz-se em parecenças nos comportamentos adotados pelos elementos do casal (e.g., ambos tentam evitar ou ambos tentam discutir um problema; Futris, Campbell, Nielsen, \& Bruwell, 2010).

Os padrões de comunicação construtiva mútua e evitamento mútuo têm sido os mais investigados
(Feeney \& Karantzas, 2017; Futris et al., 2010). O primeiro refere-se a comportamentos positivos (e.g., expressão de sentimentos, negociação) que promovem a colaboração dos elementos do casal, potenciando a resolução de problemas e estimulando diálogo, respeito, confiança e compreensão (Burrell, Kartch, Allen, \& Hill, 2014; Crenshaw, Christensen, Baucom, Epstein, \& Baucom, 2016; Feeney \& Karantzas, 2017). Assim, constitui uma resposta adaptativa ao conflito, estando associada positivamente à satisfação conjugal (Baucom, Baucom, \& Christensen, 2015; Futris et al., 2010) e sendo significativamente menos frequente em casais que procuram terapia conjugal ou optam pelo divórcio, por comparação a casais em relações estáveis (Christensen \& Shenk, 1991).

O segundo padrão descreve o processo em que os dois elementos da relação evitam totalmente o conflito, usando estratégias como o afastamento ou silêncio (Crenshaw et al., 2016). Embora este comportamento possa, a curto prazo, diminuir a intensidade do conflito, não permite a sua resolução (Feeney \& Karantzas, 2017). De forma congruente, quanto mais acentuada é a tendência dos indivíduos para evitar experiências conjugais negativas (e.g., conflitos), maior é o número de problemas conjugais existentes e pior é a perceção sobre o apoio recebido do companheiro (Bernecker, Ghassemi, \& Brandstatter, 2019).

\section{Padrões de Comunicação Complementares}

A complementaridade ou assimetria de papéis refere-se às diferenças entre os comportamentos dos elementos do casal, que se complementam (e.g., um dos elementos faz acusações e o outro defende-se; Futris et al., 2010; Sullaway \& Christensen, 1983). Por norma, esta interação é baseada nas diferenças de poder entre os elementos do casal (Watzlawick, 2014).

$\mathrm{Na}$ investigação, tem sido dado destaque ao padrão exigir/afastar-se (E/A; e.g., Schrodt et al., 2014). Os comportamentos de exigência envolvem acusações, ataques, reclamações e críticas, associando-se ao desejo de mudança, proximidade e intimidade (Eldridge \& Baucom, 2012). Os comportamentos de afastamento, por sua vez, constituem um esforço para evitar mudanças, através da mudança de assunto ou da 
recusa em responder (Eldridge \& Baucom, 2012), associando-se maioritariamente à falta de compromisso (Christensen, 1988; Christensen \& Heavey, 1990). Não obstante, podem também constituir uma tentativa de evitar o conflito, para proteção dos laços afetivos e do relacionamento (Liu \& Roloff, 2015). A polarização dos comportamentos ocorre quando os desejos (e.g., proximidade, intimidade, mudança e autonomia) dos elementos do casal são díspares (Eldridge \& Baucom, 2012). Nesse contexto, o comportamento de um dos elementos intensifica $o$ do companheiro, acentuando cada vez mais a assimetria entre ambos (e.g., quanto maior a exigência de um elemento, maior o afastamento do outro; Eldridge et al., 2017) e potenciando a ativação emocional em ambos (Baucom, Dickenson, et al., 2015).

Este padrão é característico de interações conjugais marcadas por conflito e está frequentemente presente em casais que procuram terapia conjugal (Eldridge \& Baucom, 2012; Schrodt et al., 2014), visto prejudicar o funcionamento da relação. Especificamente, este padrão, potenciado pela frustração de um ou ambos os elementos do casal (Vanhee, Lemmes, Stas, Loyes, \& Verhofstad, 2018), associa-se negativamente à comunicação construtiva (Pickover, Dobson, Tran, Lipinski, \& Beck, 2019) e positivamente à insatisfação conjugal, à insatisfação com a comunicação (Burrell et al., 2014) e ao divórcio (Christensen \& Eldridge, 2009; Ebrahimia \& Kimiaei, 2014). Adicionalmente, associa-se positivamente a comportamentos de violência conjugal (física e psicológica) exercidos pela pessoa que exige (Pickover et al., 2019). Atendendo a isto, este padrão é considerado dos mais destrutivos para as relações interpessoais, independentemente de serem as mulheres a exigir e os homens a afastarse ou o inverso (Schrodt et al., 2014). No que toca a indicadores individuais, este padrão também se mostra desadaptativo, associando-se positivamente a sintomatologia depressiva (Holley, Haase, Chui, \& Bloch, 2018; Li \& Johnson, 2018). Além das implicações que trazem aos elementos do casal, os comportamentos característicos do padrão E/A têm potencial para ser modelados pelos adultos, que poderão influenciar os filhos a adotar os mesmos comportamentos, tendencialmente também aprendidos no contexto familiar precoce dos adultos (Burrell et al., 2014).

Vários investigadores têm feito comparações de género neste padrão comunicacional, concluindo que, independentemente da orientação sexual (Baucom, McFarland, \& Christensen, 2010), as mulheres tendem mais a exigir, enquanto os homens tendem maioritariamente a afastar-se (Eldridge et al., 2017). As primeiras explicações avançadas para este resultado incluíam abordagens essencialistas, que pressupõem diferenças estáveis entre os géneros, decorrentes de fatores biológicos (maior dificuldade, nos homens, de tolerar a ativação fisiológica aversiva associada ao conflito) e/ou sociais (maior valorização da intimidade pelas mulheres e da autonomia pelo homens; Eldridge et al., 2017), que não explicariam a existência do padrão E/A em casais homossexuais (Holley, Sturm, \& Levenson, 2010). Alternativamente, propõe-se que os elementos do casal podem estar em posições diferentes no que toca ao poder que têm na relação. No caso das relações heterossexuais, a posição masculina é tendencialmente mais favorável (e.g., menor sobrecarga em resultado da divisão desequilibrada das tarefas domésticas; Knight \& Alberts, 2018), o que potencia maior desejo de mudança nas mulheres, ocorrendo o inverso nos homens. De forma congruente, o desejo de mudança associa-se positivamente ao padrão E/A (Holley et al., 2010).

\section{Avaliação dos padrões de comunicação em casais}

O método observacional é frequentemente usado para avaliar interações conjugais. No entanto, este tem diversas limitações, incluindo custos, tempo elevado de avaliação, falta de espontaneidade inerente à avaliação laboratorial (e.g., quando é pedido aos casais que se envolvam numa discussão durante a qual são filmados) e o facto de a codificação dos investigadores sobre a interação conjugal poder não traduzir a perceção dos elementos do casal (Futris et al., 2010). Além disso, as medidas de autorrelato existentes à data do desenvolvimento do Questionário de Padrões de Comunicação (QPC) não focavam sequências interacionais, privilegiando o conteúdo da comunicação conjugal, bem como comportamentos 
Quadro 1. Estruturas Fatoriais Propostas para o QPC-VC

\begin{tabular}{|c|c|c|c|c|c|}
\hline Itens originais & $\mathrm{M} 1^{\mathrm{a}}$ & $\mathrm{M} 2^{\mathrm{b}}$ & $\mathrm{M}^{\mathrm{c}}$ & $\mathrm{M} 4^{\mathrm{d}}$ & $\mathrm{M}^{\mathrm{e}}$ \\
\hline 1. Both my partner and I avoid discussing the problem. & & & $\mathrm{E} / \mathrm{A}$ & $\mathrm{E} / \mathrm{A}$ & E/A \\
\hline 2. Both my partner and I try to discuss the problem. & IPG & IPG & IPG & IPG & IPG \\
\hline $\begin{array}{l}\text { 3. I try to start a discussion while my partner tries to avoid a } \\
\text { discussion. }\end{array}$ & $\mathrm{ME} / \mathrm{HA}$ & T E/A & $\mathrm{E} / \mathrm{A}$ & $\mathrm{E} / \mathrm{A}$ & $\mathrm{E} / \mathrm{A}$ \\
\hline $\begin{array}{l}\text { 4. My partner tries to start a discussion while I try to avoid a } \\
\text { discussion }\end{array}$ & HE/MA & T E/A & $\mathrm{E} / \mathrm{A}$ & $\mathrm{E} / \mathrm{A}$ & $\mathrm{E} / \mathrm{A}$ \\
\hline 5. Both my partner and I express our feelings to each other. & IPG & IPG & IPG & IPG & IPG \\
\hline 6. Both my partner and I blame, accuse, and criticize one another. & & & $\mathrm{C} / \mathrm{D}$ & $\mathrm{C} / \mathrm{D}$ & $\mathrm{C} / \mathrm{D}$ \\
\hline $\begin{array}{l}\text { 7. Both my partner and I suggest possible solutions and } \\
\text { compromises }\end{array}$ & IPG & IPG & IPG & IPG & IPG \\
\hline $\begin{array}{l}\text { 8. I nag and demand while my partner withdraws, becomes silent, } \\
\text { or refuses to discuss the matter further. }\end{array}$ & $\mathrm{ME} / \mathrm{HA}$ & T E/A & $\mathrm{E} / \mathrm{A}$ & $\mathrm{C} / \mathrm{D}$ e $\mathrm{E} / \mathrm{A}$ & \\
\hline $\begin{array}{l}\text { 9. My partner nags and demands while I withdraw, become silent, } \\
\text { or refuse to discuss the matter further. }\end{array}$ & $\mathrm{HE} / \mathrm{MA}$ & T E/A & $\mathrm{E} / \mathrm{A}$ & $\mathrm{C} / \mathrm{D}$ e $\mathrm{E} / \mathrm{A}$ & \\
\hline 10. I criticize while my partner defends himself or herself. & $\mathrm{ME} / \mathrm{HA}$ & $\mathrm{T} \mathrm{E} / \mathrm{A}$ & $\mathrm{C} / \mathrm{D}$ & $\mathrm{C} / \mathrm{D}$ & $\mathrm{C} / \mathrm{D}$ \\
\hline 11. My partner criticizes while I defend myself. & HE/MA & $\mathrm{T} \mathrm{E} / \mathrm{A}$ & $\mathrm{C} / \mathrm{D}$ & $\mathrm{C} / \mathrm{D}$ & $\mathrm{C} / \mathrm{D}$ \\
\hline
\end{tabular}

Nota. $\quad \mathrm{C} / \mathrm{D}=$ =Criticar/Defender; $\quad$ E/A=Exigir/Afastar; $\quad$ IPG=Interações $\quad$ Positivas $\quad$ Gerais; HE/MA=Homem Exige/Mulher Afasta-se; ME/HA=Mulher Exige/Homem Afasta-se; T E/A=Total Exigir/Afastar-se. a Modelo 1 (Christensen e Heavey, citados em Futris et al., 2010): 9 itens organizados em três fatores: IPG, ME/HA e ME/HA. b Modelo 2 (Christensen e Heavey, citados em Futris et al., 2010): 9 itens organizados em dois fatores: T E/A e IPG. c Modelo 3 (Futris et al., 2010): 11 itens organizados em três fatores: IPG, E/A e C/D. d Modelo 4 (Futris et al., 2010 ): 11 itens - itens 8 e 9 com dupla saturação organizados em três fatores: IPG, E/A e C/D. e Modelo 5 (Futris et al., 2010): 9 itens - itens 8 e 9 retirados - organizados em três fatores: IPG, E/A e C/D."

e reações específicas dos dois elementos (Christensen, 1988).

Assim, o desenvolvimento do QPC decorreu da intenção de colmatar as limitações e lacunas previamente referidas, pretendendo-se criar uma medida de baixo custo e simples das interações típicas dos casais (Christensen, 1988). Esta medida tornou-se uma das formas mais comuns de avaliar o padrão E/A (Schrodt et al., 2014). Ao elaborar os itens do QPC, Christensen e Sullaway consideraram a sua experiência clínica, bem como os resultados de investigações prévias (e.g., Gottman, 1979). A versão de 35 itens permite analisar os padrões comunicacionais dos casais em três momentos: quando surge um conflito, durante o conflito e após o conflito terminar (Futris et al., 2010).

Foram propostas diversas estruturas fatoriais para o QPC, estando a complementaridade e/ou simetria de papéis na base de todas (Futris et al., 2010). Primeiramente, Christensen (citado em Futris et al., 2010) propôs uma estrutura com três subescalas: Comunicação E/A (um dos elementos do casal inicia a discussão, exige, critica e reclama, enquanto o outro evita ou se afasta), Papéis E/A (identificando os padrões de género no padrão E/A) e Comunicação Construtiva Mútua. Mais tarde, Christensen e Shenk (1991) estruturaram o instrumento em três subescalas: duas subescalas de padrões simétricos (Comunicação Construtiva Mútua e Evitamento Mútuo) e uma subescala de padrões complementares
(Comunicação E/A, integrando dois padrões específicos: Mulher Exige/Homem Afasta-se ME/HA - e Homem Exige/Mulher Afasta-se HE/MA). A proposta mais recente que conhecemos, de Crenshaw et al. (2016), é igualmente trifatorial: Comunicação Construtiva ( $\alpha$ entre .67 e .86), Próprio Exige/Companheiro(a) Afasta-se ( $\alpha$ entre .62 e .81) e Companheiro(a) Exige/Próprio Afasta-se ( $\alpha$ entre .73 e .82).

Em 1990, Christensen e Heavey (citados em Futris et al., 2010) produziram uma versão curta deste questionário (QPC-VC), considerando apenas os dois primeiros períodos temporais previamente avaliados. Dos 11 itens, seis avaliam padrões complementares (discussão/ evitamento, crítica/defesa e exigência/afastamento) e os restantes focam padrões simétricos (discussão mútua, expressão de sentimentos, negociação, evitamento e culpa). Estes autores propuseram uma cotação assente em quatro subescalas (excluindo os itens 1 e 6): ME/HA; HE/MA; Total E/A (correspondendo à soma das duas subescalas anteriores); e Interações Positivas Gerais (IPG; cf. Quadro 1).

Futris et al. (2010) propuseram uma estrutura fatorial alternativa com três subescalas: Criticar/Defender (C/D), E/A e IPG. A primeira subescala foi incluída por focar características que originam e mantêm o conflito, sem constituir um padrão de destruição que gere afastamento emocional e/ou físico da interação. Esta distinção foi feita por Gottman (1994), que ressalva que, embora ambos os padrões sejam disfuncionais e 
preditores de separação conjugal, perante defensividade - uma reação frequente ao criticismo - a interação entre os elementos do casal tende a manter-se, enquanto o afastamento, seja emocional, psicológico e/ou físico, potencia o fim da interação, sendo mais grave do ponto de vista clínico.

O estudo de Futris et al. (2010) constitui a mais recente avaliação das propriedades psicométricas do QPC-VC de que temos conhecimento. Através de análises fatoriais confirmatórias, foram comparados cinco modelos (cf. Quadro 1). Os modelos 1 e 2, propostos pelos autores originais do QPC-VC, não foram suportados. Os modelos 3 (estrutura trifatorial com cada item associado apenas a um fator) e 4 (estrutura trifatorial, com os itens 8 e 9 associados a dois fatores), propostos por Futris et al. (2010), revelaram-se adequados. Na subescala IPG, a consistência interna foi indesejável $(\alpha=.61)$; sendo respeitável $(\alpha=.71, \mathrm{E} / \mathrm{A})$ ou muito boa $(\alpha=.83$, C/D) nas restantes subescalas (DeVellis, 2017; Futris et al., 2010). Adicionalmente, todas as subescalas permitiram diferenciar pessoas com ajustamento diádico baixo e elevado (Futris et al., 2010).

Considerando a relevância dos padrões de comunicação para as relações conjugais, e dado o facto de o QPC-VC ser um instrumento breve, fácil de usar e com utilidade clínica, o presente estudo teve como objetivo avaliar as características psicométricas da versão portuguesa do QPC-VC.

\section{Método}

\section{Procedimento}

O presente estudo foi realizado após a aprovação da Comissão de Ética e Deontologia para a Investigação Científica da [Universidade Lusófona de Humanidades e Tecnologias]. O estudo teve um desenho transversal e observacional.

Primeiramente, contactámos Andrew Christensen, um dos autores da versão original do QPC, que autorizou a adaptação do questionário para português. O QPC-VC foi traduzido por duas psicólogas com experiência na adaptação de instrumentos: cada uma traduziu o instrumento separadamente; posteriormente, compararam as duas versões e desenvolveram a versão final do questionário.

O protocolo de avaliação do estudo foi disponibilizado numa plataforma de preenchimento online (Typeform), cujo link foi divulgado através de redes sociais, entre outubro de 2018 e janeiro de 2019. Recrutámos uma amostra de conveniência. Todas as pessoas que aceitaram participar no estudo leram, previamente, o consentimento informado. Não foram recolhidos dados que permitissem a identificação dos participantes. O preenchimento do protocolo de avaliação demorou, em média, 20 minutos.

\section{Participantes}

$\mathrm{Na}$ concetualização original dos itens, um determinado fator (e.g., ME/HA) é composto por diferentes itens, consoante estes sejam respondidos por mulheres heterossexuais (e.g., 4 , 9 e 11) ou homens heterossexuais (e.g., 3, 8, e 10; cf. Quadro 1), pelo que a interpretação do item depende de quem o preenche. Por exemplo, o item 10 ("Durante uma discussão sobre um problema na nossa relação, eu critico, enquanto o/a meu/minha companheiro/a se defende") pertencerá ao fator "ME/HA" caso seja respondido por uma mulher heterossexual e ao fator "HE/MA" caso seja respondido por um homem heterossexual. Isto impossibilita uma análise fatorial conjunta das respostas de homens e mulheres. Desta forma, o presente estudo focouse apenas em mulheres. Adicionalmente, foram considerados os seguintes critérios de inclusão: 1) estar num relacionamento amoroso heterossexual, 2) ter 18 anos ou mais e 3 ) ter nacionalidade portuguesa.

A amostra incluiu 164 mulheres, maioritariamente do distrito de Lisboa $(72,6 \%$, $n=119)$. As participantes apresentaram, em média, 31,63 anos de idade $(D P=9,02)$ e 14,98 anos de escolaridade $(D P=3,36)$. A maioria encontrava-se empregada $(n=111 ; 67,7 \%), 15,9 \% \quad(n=26)$ eram estudantes, $8,5 \% \quad(n=14)$ eram trabalhadorasestudantes, 7,3\% ( $n=12)$ estavam desempregadas e uma pessoa estava reformada. Treze por cento $(n=29)$ tinham diagnóstico de doença física ou psiquiátrica e 18,8\% (n=43) já tinham tido acompanhamento psicológico ou psiquiátrico.

Relativamente ao relacionamento amoroso, a 
duração média foi de 5 anos $(D P=6,75)$, variando de 1 mês a 34 anos. Setenta e dois por cento $(n=118)$ das participantes viviam com o companheiro e $62 \%(n=101)$ não tinham filhos. Menos de dois por cento $(n=3)$ da amostra já tinha feito terapia conjugal.

\section{Medidas}

Questionário sociodemográfico e clínico. Recolhemos informações sociodemográficas (e.g., sexo, idade, nacionalidade), relacionais (e.g., duração da relação) e clínicas (e.g., história médica).

QPC-VC (Christensen \& Heavey, citados em Futris et al., 2010). A escala contém 11 itens (cf. Quadro 1) e uma escala de Likert de 9 pontos, sendo solicitado aos participantes que indiquem a probabilidade de interagir com o/a companheiro/a de determinada maneira ao discutir um problema na relação (de 1 - Muito improvável a 9 - Muito provável).

Questionário de Estilos Afetivos (QEA; Hofmann \& Kashdan, 2010; Leal, 2020). Questionário de 20 itens que avaliam a regulação emocional através de uma escala de Likert de 5 pontos (de 1 - Nada verdadeiro para mim a 5 Extremamente verdadeiro para mim). As três subescalas avaliam diferentes estilos afetivos: Ocultar (e.g., "Geralmente, as pessoas não conseguem notar quando estou triste"), Ajustar (e.g., "Consigo acalmar-me muito rapidamente") e Tolerar (e.g., "Não há problema em, por vezes, sentir emoções negativas"). Os itens de cada fator são somados, com pontuações mais elevadas a refletir preferência por um dado estilo afetivo. As duas primeiras subescalas têm apresentado uma consistência interna muito boa ( $\alpha$ entre .80 e .84), sendo esta indesejável para a subescala Tolerar $(\alpha=.66)$. Este padrão também se verificou na amostra do estudo, sendo o alfa de Cronbach de .60 (Tolerar; esta subescala não foi utilizada); .79 (Ajustar) e .84 (Ocultar).

Escala de Ajustamento Diádico - Revista (EAD-R; Busby, Christensen, Crane, \& Larson, 1995; Pereira, Moura-Ramos, Narciso, \& Canavarro, 2017). É composta por 14 itens que avaliam três dimensões do ajustamento na relação conjugal: Consenso (e.g., grau de acordo dos elementos do casal em tomadas de decisão importantes), Satisfação (e.g., frequência com que o casal discute) e Coesão (e.g., frequência com que o casal tem uma troca de ideias estimulante). A escala de resposta é de tipo Likert de 5 ou 6 pontos, consoante os itens. A pontuação total varia entre 0 e 69; quanto mais elevada, maior a satisfação com a relação conjugal. Na amostra do estudo, os valores de consistência interna foram adequados (Escala total: $\alpha$.85; Consenso: $\alpha=.75$; Satisfação: $\alpha$.77; Coesão: $\alpha=.75$ ).

\section{Análises estatísticas}

Todas as análises estatísticas foram realizadas no IBM SPSS Statistics 25. As análises incluíram: análise de componentes principais (ACP) com rotação oblíqua Promax (dada a previsão de que os fatores estariam correlacionados; Marôco, 2018), para avaliar a validade fatorial (Costello \& Osborne, 2005); coeficientes de correlação de Pearson com uma medida de um construto teoricamente relacionado (ajustamento na relação conjugal) para avaliar a validade convergente e com uma medida de um construto teoricamente distinto (estilos afetivos) para avaliar a validade divergente; alfa de Cronbach e correlações itemtotal corrigidas, para analisar a fidelidade; e estatísticas descritivas e o teste de KolmogorovSmirnov, para avaliar a distribuição e sensibilidade dos fatores e dos itens do QPC-VC.

\section{Resultados}

\section{Validade Fatorial}

Relativamente aos pressupostos para a realização da ACP, o número de participantes do estudo é superior ao mínimo recomendado (10 participantes por item, correspondendo, neste caso, a 110 respostas; Pallant, 2016). Adicionalmente, o índice Kaiser-Meyer-Olkin (.81) e o teste de esfericidade de Bartlett (796,126, $p<.05)$ demonstraram que existem relações suficientemente fortes entre as variáveis do QPCVC (Howard, 2016).

De acordo com o critério de Kaiser, são extraídos fatores com valores próprios superiores a 1 (Field, 2018). Recorrendo à análise paralela, o número de fatores a extrair corresponde ao número de fatores cujo valor próprio dos dados reais é superior aos valores próprios dos 100 
Quadro 2. Valores de Saturação e Comunalidades dos Itens do QPC-VC

\begin{tabular}{|c|c|c|c|c|}
\hline \multirow{2}{*}{ Item } & \multicolumn{3}{|c|}{ Valores de saturação } & \multirow{2}{*}{ Comunalidades } \\
\hline & 1 & 2 & 3 & \\
\hline $\begin{array}{l}\text { 5. Tanto eu como o meu companheiro expressamos os nossos } \\
\text { sentimentos um ao outro. }\end{array}$ & .86 & -.36 & -.09 & .74 \\
\hline $\begin{array}{l}\text { 7. Tanto eu como o meu companheiro sugerimos possíveis soluções e } \\
\text { cedências. }\end{array}$ & .82 & -.39 & -.10 & .68 \\
\hline 2. Tanto eu como o meu companheiro tentamos discutir o problema. & .81 & -.29 & -.34 & .73 \\
\hline 1. Tanto eu como o meu companheiro evitamos discutir o problema. & -.80 & .32 & .22 & 67 \\
\hline 11. O meu companheiro critica, enquanto eu me defendo. & -.29 & .86 & .29 & .78 \\
\hline 10. Eu critico, enquanto o meu companheiro se defende. & -.31 & .79 & .17 & .63 \\
\hline $\begin{array}{l}\text { 6. Tanto eu como o meu companheiro nos culpamos, acusamos e } \\
\text { criticamos um ao outro. }\end{array}$ & -.22 & .77 & .28 & .64 \\
\hline $\begin{array}{l}\text { 3. Eu tento iniciar uma discussão sobre o problema, enquanto o meu } \\
\text { companheiro tenta evitá-la. }\end{array}$ & -.50 & .67 & -.14 & .57 \\
\hline $\begin{array}{l}\text { 8. Eu reclamo e faço exigências, enquanto o meu companheiro se } \\
\text { afasta, fica calado ou se recusa a continuar a discutir o assunto. }\end{array}$ & -.54 & .67 & -.20 & .63 \\
\hline $\begin{array}{l}\text { 4. O meu companheiro tenta iniciar uma discussão sobre o problema, } \\
\text { enquanto eu tento evitá-la. }\end{array}$ & -.23 & .11 & .83 & .71 \\
\hline $\begin{array}{l}\text { 9. O meu companheiro reclama e faz exigências, enquanto eu me } \\
\text { afasto, fico calada ou me recuso a continuar a discutir o assunto. }\end{array}$ & -.29 & .42 & .81 & .76 \\
\hline
\end{tabular}

Nota. A negrito estão apresentados os valores de saturação mais elevados para cada item.

Quadro 3. Coeficientes de Correlação entre o QPC-VC, a EAD-R e o QEA

\begin{tabular}{lccc}
\hline Itens & PSP & PAN & PCN \\
\hline EAD-R_Escala total & $.54 * * *$ & $-.50 * * *$ & $-.31 * * *$ \\
EAD-R_Consenso & $.42 * * *$ & $-.39 * * *$ & $-.22^{* * *}$ \\
EAD-R_Satisfação & $.43 * * *$ & $-.55 * * *$ & $-.32 * * *$ \\
EAD-R_Coesão & $.48^{* * *}$ & $-.35 * * *$ & $-.23 * *$ \\
QEA_Ocultar & $-.22 * *$ & .03 & -.00 \\
QEA_Ajustar & $.21 * *$ & $-.19 *$ & -.07 \\
\hline
\end{tabular}

Nota. EAD-R=Escala de Ajustamento Diádico-Revista; PAN=Padrão Alternado Negativo; PCN=Padrão Complementar Negativo; $\mathrm{PSP}=$ Padrão Simétrico Positivo; QEA=Questionário de Estilos Afetivos.

$* p<.05 ; * * p<.01 ; * * * p<.001$.

conjuntos de dados aleatórios da análise paralela, considerando a média e o percentil 95 (Fabrigar \& Wegener, 2012). Ambos os métodos conduziram a resultados congruentes, isto é, uma estrutura trifatorial da versão portuguesa do QPC-VC: o fator 1 explicou 40,86\% da variância total; o fator 2 explicou $15,10 \%$ da variância total; e o fator 3 explicou $12,92 \%$ da variância total, sendo que a variância total explicada cumpre o mínimo recomendado de 50\% (Meyers, Gamst, \& Guarino, 2017).

Apesar de os itens 3, 8 e 9 apresentarem valores de saturação superiores a $0,40 \mathrm{em}$ dois fatores (cf. Quadro 2), não foram excluídos, por duas razões: 1) cada fator deve incluir, no mínimo, três ou quatro itens; e 2) as comunalidades dos itens devem ser iguais ou superiores a 0,50 (Meyers et al., 2017), o que se verificou. Estes três itens foram incluídos no fator em que apresentaram valores de saturação mais altos, auxiliando a interpretação desse mesmo fator.

Considerando as concetualizações anteriores da escala (cf. Quadro 1), apesar de algumas semelhanças, não há sobreposição total na estrutura fatorial. Assim, propomos novas designações para os fatores: Padrão Simétrico Positivo (PSP; itens 1 invertido, 2, 5 e 7); Padrão Alternado Negativo (PAN; itens 3, 6, 8, 10 e 11) e Padrão Complementar Negativo (PCN; itens 4 e 9).

Os fatores do QPC-VC correlacionaram-se: PSP associou-se negativamente ao PCN ( $r=-.45$, $p<.001)$ e ao PAN $(r=-.34, p<.001)$, com um efeito médio a grande; os padrões negativos associaram-se positivamente $(r=.29, p<.001)$, com um efeito pequeno (Meyers et al., 2017).

\section{Validade Convergente}

As escalas da EAD-R correlacionam-se negativamente com os fatores PAN e PCN e positivamente com o fator PSP. O tamanho do efeito dessas correlações variou de pequeno a grande (cf. Quadro 3).

\section{Validade Divergente}

As duas subescalas do QEA usadas não se correlacionaram com o QPC-VC ou, quando existiram correlações, foram de efeito pequeno (cf. Quadro 3). 
Quadro 4. Correlações Item-Total Corrigidas e Alfa de Cronbach Excluindo cada Item para o QPC-VC

\begin{tabular}{lccc}
\hline Fatores & Itens & Correlação item-total corrigida & Alfa de Cronbach excluindo o item \\
\hline PSP & 1 & .68 & .83 \\
& 2 & .71 & .82 \\
& 5 & .75 & .80 \\
& 7 & .69 & .83 \\
PAN & 3 & .55 & .79 \\
& 6 & .58 & .78 \\
& 8 & .55 & .78 \\
& 10 & .61 & .77 \\
& 11 & .70 & .74 \\
PCN & 4 & .54 &. \\
& 9 & .54 &. \\
\hline
\end{tabular}

Nota. PAN=Padrão Alternado Negativo; PCN=Padrão Complementar Negativo; PSP=Padrão Simétrico Positivo.

Quadro 5. Estatísticas Descritivas dos Itens e Fatores do QPC-VC

\begin{tabular}{lcccc}
\hline Item / Fator & $M(D P)$ & Assimetria & Curtose & Kolmogorov-Smirnov \\
\hline 1 & $3.05(2.13)$ & 1.02 & .17 & $.20^{* * * *}$ \\
2 & $7.28(1.86)$ & -1.41 & 1.79 & $.21^{* * *}$ \\
3 & $4.02(2.82)$ & .40 & -1.28 & $.18^{* * * *}$ \\
4 & $2.85(2.19)$ & 1.13 & .35 & $.23^{* * *}$ \\
5 & $7.14(2.02)$ & -1.17 & .88 & $.19^{* * *}$ \\
6 & $3.96(2.63)$ & .45 & -1.05 & $.15^{* * *}$ \\
7 & $7.00(1.90)$ & -1.08 & .77 & $.19^{* * *}$ \\
8 & $3.17(2.45)$ & .95 & -.29 & $.21^{* * *}$ \\
9 & $2.75(2.09)$ & 1.21 & .54 & $.22^{* * *}$ \\
10 & $3.55(2.40)$ & .55 & -.88 & $.17^{* * *}$ \\
11 & $3.46(2.34)$ & .59 & -.65 & $.19^{* * *}$ \\
& & & & $.13^{* * *}$ \\
PSP & $7.09(1.66)$ & -1.14 & 1.47 & $.08^{* *}$ \\
PAN & $3.63(1.91)$ & .24 & -1.04 & $.17^{* * * *}$ \\
\hline
\end{tabular}

Nota. PAN=Padrão Alternado Negativo; PCN=Padrão Complementar Negativo; PSP=Padrão Simétrico Positivo.

$* * p<.01 ; * * * p<.001$.

\section{Fidelidade}

Relativamente à consistência interna, esta foi muito boa para os fatores PSP $(\alpha=.86)$ e PAN $(\alpha=.81)$ e respeitável para o fator $\operatorname{PCN}(\alpha=.70$; DeVellis, 2017).

Todos os itens apresentaram uma correlação item-total corrigida acima de .50, indicando uma boa correlação com o construto medido pelos restantes itens (cf. Quadro 4; Finch, Immekus, \& French, 2016). Relativamente ao alfa de Cronbach se o item fosse excluído, a consistência interna de cada fator diminuiria em todos os casos.

\section{Sensibilidade}

A distribuição das respostas aos itens do QPC-VC diferiu da normal (cf. Quadro 5; Field, 2018). Relativamente à assimetria e curtose, foi utilizado o critério liberal, considerando-se desejável que os valores oscilem entre -1 e 1 (Meyers et al., 2017). Os itens 2, 5 e 7 apresentaram uma distribuição assimétrica à esquerda e os itens 1, 4 e 9 uma distribuição assimétrica à direita. No que respeita à curtose, os itens 3 e 6 apresentaram uma distribuição platicúrtica e o item 2 uma distribuição leptocúrtica (cf. Quadro 5; Marôco, 2018).

Nos fatores, a distribuição das respostas diferiu da normal (cf. Quadro 5; Field, 2018). Relativamente à assimetria, o fator PSP apresentou uma distribuição assimétrica à esquerda e o fator PCN uma distribuição assimétrica à direita. No que respeita à curtose, o fator PSP apresentou uma distribuição leptocúrtica e o fator PAN uma distribuição platicúrtica (Marôco, 2018).

\section{Discussão}

$\mathrm{O}$ nosso estudo analisou a estrutura fatorial e as características psicométricas do QPC-VC numa amostra de mulheres portuguesas heterossexuais. Os modelos originais de Christensen e Heavey e os modelos alternativos desenvolvidos por Futris et al. (2010), apesar de apresentarem algumas 
semelhanças com a estrutura que obtivemos, não se revelaram totalmente adequados para esta amostra portuguesa. Assim, propomos uma estrutura trifatorial, incluindo um padrão positivo (PSP) e dois padrões negativos (PAN e PCN), todos adequadamente fiáveis e válidos.

Relativamente às designações dos fatores, foram escolhidas tendo em conta o tipo de padrões de comunicação (i.e., complementares ou simétricos) e o seu impacto na relação conjugal (i.e., negativo ou positivo). De acordo com todas as concetualizações anteriores do QPC-VC (cf. Quadro 1), os padrões de comunicação positivos simétricos incluem a discussão do problema, a expressão de sentimentos e a negociação. No nosso estudo, esta subescala incluiu um comportamento simétrico adicional: o evitamento da discussão (previamente incluído no padrão E/A; Futris et al., 2010). Dado que este item se correlacionou negativamente com os restantes itens da subescala, consideramos que representa um comportamento positivo, visto que uma baixa tendência para o evitamento do conflito se tem revelado adaptativa (e.g., Bernecker et al., 2019). É de salientar que, no presente estudo, a consistência interna desta subescala foi notoriamente superior à que Futris et al. (2010) obtiveram para a IPG composta por três itens (.86 vs. .61), constituindo um indicador adicional de que a composição portuguesa do PSP é mais adequada do que a original.

$\mathrm{O}$ fator PAN englobou itens que, originalmente, foram concetualizados como sendo característicos dos padrões ME/HA (itens 3, 8 e 10) e HE/MA (item 11) e, nos modelos de Futris et al. (2010), como específicos do padrão E/A (itens 3 e 8) e do padrão C/D (item 6, 10 e 11; Futris et al., 2010). Estudos anteriores demonstraram que as pessoas reagem ao comportamento do companheiro, o que promove a escalada mútua no ciclo de interações negativas (Baucom, Dickenson, et al., 2015). No mesmo sentido, as exigências, feitas maioritariamente por mulheres (Eldridge et al., 2017), tendem a dar início a um padrão bidirecional, com as influências mútuas entre os elementos do casal a resultar em múltiplas exigências e afastamentos, formando uma sequência interacional que decorre ao longo do tempo (Eldridge \& Baucom, 2012; Vanhee et al., 2018; Winstok \& Smadar-Dror,
2018). No entanto, esta sequência não é necessariamente homogénea, podendo haver alternância entre comportamentos complementares (e.g., num determinado momento, os homens exigem e as mulheres afastam-se ou vice-versa, dependendo do decurso da comunicação e do que está a ser debatido) e comportamentos simétricos (e.g., expressão de acusações mútuas), sendo que todos estimulam o conflito (Watzlawick, 2014). Deste modo, concluímos que o fator PAN traduz uma sequência interacional de escalada do conflito (Winstok \& Smadar-Dror, 2018), em que os elementos do casal podem assumir padrões de comunicação complementares e/ou simétricos.

Por fim, o fator PCN revelou-se semelhante aos modelos originais e alternativos (cf. Quadro 1), definindo-se por um padrão de comunicação complementar, onde predominantemente os homens exigem e as mulheres se afastam, o que também promove insatisfação conjugal (Schrodt et al., 2014). A média desta subescala foi inferior à do PAN, o que é congruente com o facto de, tendencialmente, os homens demonstrarem comportamentos de exigência com menos frequência do que as mulheres (Eldridge et al., 2017).

Por fim, os resultados das correlações foram ao encontro das expetativas, demonstrando o impacto diferenciado de cada um destes padrões comunicacionais. O PSP denota ser adaptativo, associando-se negativamente aos restantes dois padrões e ao estilo afetivo Ocultar (um estilo potencialmente desadaptativo, dada a sua associação positiva com sintomatologia depressiva e ansiosa; Leal, 2020) e positivamente ao ajustamento diádico e ao estilo afetivo Ajustar (cujo carácter adaptativo se traduz nas consistentes associações negativas com a psicopatologia; Leal, 2020). Os padrões que representam comportamentos negativos apresentaram correlações negativas com o ajustamento diádico, reforçando o seu carácter prejudicial para a relação conjugal (Eldridge et al., 2017).

\section{Implicações Clínicas}

A comunicação entre os elementos do casal constitui um dos elementos comportamentais que devem ser considerados no âmbito de uma 
avaliação dos vários domínios associados ao sofrimento conjugal (Balderrama-Durbin, Snyder, Heyman, \& Haynes, 2020), dado que o sucesso da terapia conjugal depende da promoção da comunicação eficaz entre os elementos do casal (Benson, McGinn, \& Christensen, 2012). Dada a sua facilidade de utilização, aliada às suas características psicométricas adequadas, o QPCVC constitui uma medida adequada neste contexto clínico. O facto de o QPC-VC avaliar dois padrões comunicacionais disfuncionais e um padrão adaptativo comporta a vantagem adicional de potenciar duas informações relevantes para o planeamento da intervenção terapêutica: a (in)existência de dificuldades de comunicação e a (in)existência de recursos de comunicação.

Desta forma, e considerando o facto de constituírem fatores modificáveis (Schmidt, Luquet, \& Gehlert, 2016), os padrões comunicacionais podem ser um foco de intervenção clínica. Os psicólogos que trabalham com casais devem ter particular atenção a fatores individuais (e.g., idade mais elevada; Burrell et al., 2014) e relacionais (e.g., relações mais longas; Burrell et al., 2014) tendencialmente associados ao uso do padrão E/A, de maneira a identificar, tão precocemente quanto possível, pessoas em risco de manifestar comportamentos clinicamente relevantes. Nessa ótica, verifica-se um aumento do número de programas de prevenção, de natureza educacional, dirigidos a casais sem dificuldades conjugais (prevenção primária) ou em risco de as manifestar (prevenção secundária; Bradbury \& Bodenmann, 2020). Estes programas assentam, em grande parte, na promoção de competências de comunicação adaptativas, com vista a minimizar a deterioração na qualidade da relação conjugal, tendo demonstrado algum sucesso, particularmente no âmbito da prevenção secundária (Bradbury \& Bodenmann, 2020). A título de exemplo, uma intervenção assente na psicologia positiva, envolvendo múltiplos componentes e a realização de atividades diárias (e.g., partilha de emoções positivas) ao longo de quatro semanas, mostrou potenciar a melhoria de comportamentos de mutualidade (i.e., discussão mútua, compreensão e ausência de evitamento) entre os elementos do casal (Antoine, Andreotti, \& Congard, 2020). No âmbito da intervenção psicológica que ocorre na presença de sofrimento conjugal, a terapia conjugal tem-se revelado eficaz na melhoria da qualidade da comunicação conjugal (Roddy, Walsh, Rothman, Hatch, \& Doss, 2020). Para qualquer um destes tipos de intervenções, o QPC-VC constitui uma medida de avaliação do sucesso terapêutico, tendo sido já usado em estudos de eficácia e efetividade (e.g., Antoine et al., 2020; Ghavibazou, Hosseinian, \& Abdollahi, 2020).

No entanto, apenas uma minoria dos casais com dificuldades conjugais procura ajuda profissional, devido a fatores como atitudes negativas face à procura de ajuda e a crença de que a procura de ajuda implica o reconhecimento de que a relação conjugal falhou (Hubbard \& Harris, 2020). Assim, é importante que os psicólogos desenvolvam outro tipo de recursos que esta população possa utilizar, nomeadamente aqueles que as pessoas mais tendem a procurar como fonte de ajuda informal (e.g., livros de autoajuda, recursos na Internet; Stewart, Bradford, Higginbotham, \& Skogrand, 2016). Assim, sugerimos, por exemplo, elaborar materiais informativos que, tendo por base os itens do QPC$\mathrm{VC}$ e a literatura da área, ajudem os casais a identificar comportamentos potencialmente prejudiciais à relação e a desenvolver estratégias comunicacionais adaptativas.

\section{Limitações e Estudos Futuros}

O presente estudo descreveu a validação da versão portuguesa do QPC-VC considerando apenas mulheres heterossexuais. Desta forma, a primeira limitação do estudo respeita à falta de representatividade das participantes, sendo necessário explorar a estrutura fatorial do instrumento numa amostra de homens. A presente amostra também não é representativa da população portuguesa noutras características sociodemográficas, como a escolaridade. Tendo em conta que poderá haver especificidades sociodemográficas no uso (e.g., o nível socioeconómico associa-se negativamente à frequência de comportamentos de exigência e afastamento; Ross et al., 2019) e no impacto dos padrões de comunicação (e.g., o nível socioeconómico modera a relação entre o padrão E/A e a satisfação conjugal, sendo este mais prejudicial para pessoas com mais recursos económicos; Ross et al., 2019), estudos futuros 
deverão descrever e comparar grupos a estes níveis.

Em segundo lugar, há algumas características previamente identificadas como estando relacionadas com os padrões de comunicação, incluindo a presença de dificuldades conjugais clinicamente significativas (e.g., Eldridge, Sevier, Jones, Atkins, \& Christensen, 2007), orientação sexual (e.g., maior propensão de pessoas homossexuais para demonstrar padrões positivos; Kurdek, 2004) e existência de filhos (e.g., pessoas heterossexuais com filhos recorrem mais ao padrão E/A do que pessoas heterossexuais sem filhos; Kurdek, 2004). Em estudos futuros, poderse-ão recrutar participantes com estas características, de maneira a avaliar a validade discriminante do instrumento. Do mesmo modo, os padrões de comunicação são variáveis de acordo com os tópicos que os casais estão a discutir (Eldridge et al., 2007; Reblin, Sutton, Vadaparampil, Heyman, \& Ellington, 2019), sendo relevante identificar os tópicos mais associados a padrões de comunicação adaptativos e desadaptativos.

Relativamente à estrutura fatorial do instrumento, e dado que o modelo resultante do presente estudo difere de propostas anteriores (cf. Quadro 1), é importante comparar, no futuro, a adequação das diferentes opções de estrutura através de uma análise fatorial confirmatória. Adicionalmente, a estrutura fatorial da versão longa do QPC mostrou-se diferente dos estudos originais quando explorada num grupo de mulheres vítimas de violência conjugal (Pickover et al., 2019). Assim, a invariância da estrutura fatorial do QPC-VC deve igualmente ser testada.

Por fim, uma vez que a avaliação através do QPC-VC se baseia em autorrelatos e heterorrelatos, devemos ter em conta que os resultados podem ter sido influenciados pela desejabilidade social (o que tentámos minimizar através da garantia de anonimato dos dados e da recolha de dados online) e pela memória das participantes. Devemos notar, no entanto, que as medidas de autorrelato são frequentemente utilizadas na avaliação de indicadores conjugais, constituindo um bom método quando apresentam propriedades psicométricas adequadas (Balderrama-Durbin et al., 2020), como se verificou neste estudo.
Em suma, o QPC-VC parece ser adequado para avaliar, nas mulheres portuguesas heterossexuais, sequências interacionais simétricas, complementares e alternadas em contextos conjugais. Estudos futuros poderão aprofundar os resultados encontrados, de forma a possibilitar o uso do QPC-VC com mais populações e noutros contextos.

\section{Referências}

Antoine, P., Andreotti, E., \& Congard, A. (2020). Positive psychology intervention for couples: A pilot study. Stress \& Health, 36(2), 179190. doi:10.1002/smi.2925

Balderrama-Durbin, C. M., Snyder, D. K., Heyman, R. E., \& Haynes, S. N. (2020). Systematic and culturally sensitive assessment of couple distress. In K. S. Wampler, \& A. J. Blow (Eds.), The handbook of systematic family therapy (Vol. 3, pp. 27-48). Hoboken: Wiley.

Baucom, B. R., Dickenson, J. A., Baucom, D. H., Fisher, M. S., Atkins, D. C., Weutshoff, S., Hahlweg, K., \& Zimmermann, T. (2015). The interpersonal process model of demand/withdraw behavior. Journal of Family Psychology, 29, 80-90. doi:10.1037/fam0000044

Baucom, B. R., McFarland, P. T., \& Christensen, A. (2010). Gender, topic, and time in observed demand-withdraw interaction in cross- and same-sex couples. Journal of Family Psychology, 24, 233-242. doi:10.1037/a0019717

Baucom, K. J., Baucom, B. R., \& Christensen, A. (2015). Changes in dyadic communication during and after integrative and traditional behavioral couple therapy. Behaviour Research and Therapy, 65, 18-28. doi:10.1016/j.brat.2014.12.004

Benson, L. A., McGinn, M. M., \& Christensen, A. (2012). Common principles of couple therapy. Behavior Therapy, 43, 2-35. doi:10.1016/j.beth.2010.12.009

Bernecker, K., Ghassemi, M., \& Brandstatter, V. (2019). Approach and avoidance relationship goals and couples' nonverbal communication during conflict. European Journal of Social 
Psychology, 49(3),

$622-636$. doi:10.1002/ejsp.2379

Bradbury, T. N., \& Bodenmann, G. (2020). Interventions for couples. Annual Review of Clinical Psychology, 16, 99-123. doi:10.1146/annurev-clinpsy-071519-020546

Burrell, N. A., Kartch, F. F., Allen, M., \& Hill, C. B. (2014). A meta-analysis of demand/withdraw interaction patterns. In $\mathrm{N}$. A. Burrell, M. Allen, B. M. Gayle, \& R. W. Preiss (Eds.), Managing interpersonal conflict: Advances through meta-analysis (pp. 297-314). Nova Iorque: Routledge.

Busby, D. M., Christensen, C., Crane, D. R., \& Larson, J. H. (1995). A revision of the Dyadic Adjustment Scale for use with distressed and nondistressed couples: Construct hierarchy and multidimensional scales. Journal of Marital and Family Therapy, 21, 289-308. doi:10.1111/j.1752-0606.1995.tb00163.x

Christensen, A. (1988). Dysfunctional interaction patterns in couples. In P. Noller, \& M. A. Fitzpatrick (Eds.), Perspectives on marital interaction (pp. 31-52). Clevedon: Multilingual Matters.

Christensen, A., \& Eldridge, K. A. (2009). Demand-withdraw communication during couple conflict: A review and analysis. In P. Noller, \& J. A. Feeney (Eds.), Understanding marriage: Developments in the study of couple interaction (pp. 289-322). Cambridge: Cambridge University Press.

Christensen, A., \& Heavey, C. L. (1990). Gender and social structure in the demand/withdraw pattern of marital conflict. Journal of Personality and Social Psychology, 59(1), 7381. doi:10.1037//0022-3514.59.1.73

Christensen, A., \& Shenk, J. L. (1991). Communication, conflict, and psychological distance in nondistressed, clinic and divorcing couples. Journal of Consulting and Clinical Psychology, 59(3), 458-463. doi:10.1037//0022-006x.59.3.458

Costello, A. B., \& Osborne, J. W. (2005). Best practices in exploratory factor analysis: Four recommendations for getting the most from your analysis. Practical Assessment, Research \& Evaluation, 10, Artigo 7. doi:10.7275/jyj1-4868

Crenshaw, A. O., Christensen, A., Baucom, D. H.,
Epstein, N. B., \& Baucom, B. R. W. (2016). Revised scoring and improved reliability for the Communication Patterns Questionnaire. Psychological Assessment, 29(7), 913-925. doi:1037/pas0000385

DeVellis, R. F. (2017). Scale development: Theory and applications (4. ${ }^{\mathrm{a}}$ ed.). Thousand Oaks: SAGE.

Doss, B. D., Simpson, L. E., \& Christensen, A. (2004). Why do couples seek marital therapy? Professional Psychology: Research and Practice, 35, 608-614. doi:10.1037/0735-7028.35.6.608

Ebrahimi, E., \& Kimiaei, S. A. (2014). The study of the relationship among marital satisfaction, attachment styles, and communication patterns in divorcing couples. Journal of Divorce \& Remarriage, 55, 451-463. doi:10.1080/10502556.2014.931759

Eldridge, K. A., \& Baucom, B. (2012). Demandwithdrawal communication in couples: Recent development and future directions. In P. Noller, \& G. C. Karantzas (Eds.), The WileyBlackwell handbook of couples and family relationships (pp. 144-158). Chichester: Wiley-Blackwell.

Eldridge, K. A., Cencirulo, J., \& Edwards, E. (2017). Demand-withdraw patterns of communication in couple relationships. In J. Fitzgerald (Ed.), Foundations for couples' therapy: Research for the real world (pp. 112122). Nova Iorque: Routledge.

Eldridge, K. A., Sevier, M., Jones, J., Atkins, D. C., \& Christensen, A. (2007). Demandwithdraw communication in severely distressed, moderately distressed, and nondistressed couples: Rigidity and polarity during relationship and personal problem discussions. Journal of Family Psychology, 21, 218-226. doi:10.1037/0893-3200.21.2.218

Fabrigar, L. R., \& Wegener, D. T. (2012). Exploratory factor analysis. Oxford: Oxford University Press.

Feeney, J. A., \& Karantzas, G. C. (2017). Couple conflict: Insights from an attachment perspective. Current Opinion in Psychology, 13, 60-64. doi:10.1016/j.copsyc.2016.04.017

Field, A. (2018). Discovering statistics using IBM SPSS Statistics (5. ${ }^{\mathrm{a}}$ ed.). London: SAGE.

Finch, W. H., Immekus, J. C., \& French, B. F. 
(2016). Applied psychometrics using SPSS and AMOS. Charlotte: Information Age.

Futris, T. G., Campbell, K., Nielsen, R. B., \& Burwell, S. R. (2010). The Communication Patterns Questionnaire-Short Form: A review and assessment. The Family Journal, 18, 275287. doi:10.1177/1066480710370758

Ghavibazou, E., Hosseinian, S., \& Abdollahi, A. (2020). Effectiveness of narrative therapy on communication patterns for women experiencing low marital satisfaction. Australian and New Zealand Journal of Family Therapy, 41, 195-207. doi:10.1002/anzf.1405

González, L. F. V., Aragón, S. R., Díaz-Loving, R., \& Ramírez, I. M. (2012). Elaboración de una escala de permanencia en la relación de pareja. Revista Iberoamericana de Diagnóstico y Evaluación - e Avaliação Psicológica, 33(1), 199-218.

Gottman, J. M. (1979). Empirical investigations of marriage. Nova Iorque: Academic Press.

Gottman, J. M. (1994). What predicts divorce? The relationship between marital processes and marital outcomes. Nova Iorque: Psychology Press.

Hofmann, S. G., \& Kashdan, T. B. (2010). The Affective Style Questionnaire: Development and psychometric properties. Journal of Psychopathology and Behavioral Assessment, 32(2), 255-263. doi:10.1007/s10862-009-9142-4

Holley, S. R., Haase, C. M., Chui, I., \& Bloch, L. (2018). Depression, emotion regulation and the demand/withdraw pattern during intimate relationship conflict. Journal of Social and Personal Relationships, 35, 408-430. doi:10.1177/0265407517733334

Holley, S. R., Sturm, V. E., \& Levenson, R. W. (2010). Exploring the basis for gender differences in the demand-withdraw pattern. Journal of Homosexuality, 57, 666-684. doi:10.1080/00918361003712145

Howard, M. C. (2016). A review of exploratory factor analysis decisions and overview of current practices: What we are doing and how can we improve? International Journal of Human-Computer Interaction, 32, 51-62. doi:10.1080/10447318.2015.1087664

Hubbard, A., \& Harris, S. (2020). A critical review of help-seeking for couples therapy: Clinical implications and next steps. Contemporary Family Therapy, 42, 152-162. doi:10.1007/s10591-019-09521-w

Khaleque, A. (2018). Intimate relationships across the lifespan: Formation, development, enrichment, and maintenance. Santa Barbara: Praeger.

Knight, K., \& Alberts, J. K. (2018). Response thresholds and demand/withdraw communication in domestic labor conflict. Journal of Family Communication, 18, 110123. doi:10.1080/15267431.2018.1427588

Kurdek, L. A. (2004). Are gay and lesbian cohabiting couples really different from heterosexual married couples? Journal of Marriage and Family, 66, 880-900. doi:10.1111/j.0022-2445.2004.00060.x

Leal, M. (2020). Propriedades psicométricas da versão portuguesa do Questionário de Estilos Afetivos (tese de mestrado não publicada). Universidade Lusófona de Humanidades e Tecnologias, Lisboa.

Li, P.-F., \& Johnson, L. N. (2018). Couples' depression and relationship satisfaction: Examining the moderating effects of demand/withdraw communication patterns. Journal of Family Therapy, 40, S63-S85. doi:10.1111/1467-6427.12124

Liu, E., \& Roloff, M. E. (2015). To avoid or not to avoid: When emotions overflow. Communication Research Reports, 32, 332339. doi:10.1080/08824096.2015.1089849

Marôco, J. (2018). Análise estatística com o SPSS Statistics 25 (7. $^{\mathrm{a}}$ ed.). Pêro Pinheiro: ReportNumber.

Meraz, M. G., \& Palencia, A. R. (2012). Mantenimiento en la relación de pareja: Construcción y validación de dos escalas. Revista Iberoamericana de Diagnóstico y Evaluación - e Avaliação Psicológica, 34(1), 133-155.

Meyers, L. S., Gamst, G., \& Guarino, A. J. (2017). Applied multivariate research: Design and interpretation (3. ${ }^{\mathrm{a}}$ ed.). Thousand Oaks: SAGE.

Neumann, A. P., \& Wagner, A. (2015). Reasons to search for couple and family therapy: A systematic literature review. Psychologica, 58,23-39. doi:10.14195/1647-8606_58-1_2 
Nguyen, T. P., Williamson, H. C., \& Bradbury, T. N. (2017). Redefining communication in couple relationships. In J. Fitzgerald (Ed.), Foundations for couples' therapy: Research for the real world (pp. 61-70). Nova Iorque: Routledge.

Pallant, J. (2016). SPSS Survival manual (6. ${ }^{\mathrm{a}}$ ed.). Nova Iorque: Open University Press.

Pereira, M., Moura-Ramos, M., Narciso, I., \& Canavarro, M. C. (2017). Psychometric properties of the Revised Dyadic Adjustment Scale (RDAS) in a sample of couples: Testing the factorial invariance across gender. Comunicação oral apresentada na $14^{\text {th }}$ European Conference on Psychological Assessment, Lisboa.

Pickover, A. M., Dobson, T. S., Tran, H. N., Lipinski, A. J., \& Beck, J. G. (2019). Factor structure of the Communication Patterns Questionnaire in violence-exposed women. Journal of Interpersonal Violence. doi:10.1177/0886260519867147

Reblin, M., Sutton, S. K., Vadaparampil, S. T., Heyman, R. E., \& Ellington, L. (2019). Behind closed doors: How advanced cancer couples communicate at home. Journal of Psychosocial Oncology, 37, 228-241. doi:10.1080/07347332.2018.1508535

Roddy, M. K., Rothman, K., Cicila, L. N., \& Doss, B. D. (2019). Why do couples seek relationship help online? Description and comparison to in-person interventions. Journal of Marital and Family Therapy, 45(3), 369-379. doi:10.1111/jmft.12329

Roddy, M. K., Walsh, L. M., Rothman, K., Hatch, S. G., \& Doss, B. D. (2020). Meta-analysis of couple therapy: Effects across outcomes, designs, timeframes, and other moderators. Journal of Consulting and Clinical Psychology, 88, 583-596. doi:10.1037/ccp0000514

Ross, J. M., Karney, B. R., Nguyen, T. P., \& Bradbury, T. N. (2019). Communication that is maladaptive for middle-class couples is adaptive for socioeconomically disadvantaged couples. Journal of Personality and Social Psychology, 116(4), 582-597. doi: $10.1037 / \mathrm{pspi0} 000158$

Schmidt, C. D., Luquet, W., \& Gehlert, N. C. (2016). Evaluating the impact of the "Getting
The Love You Want" couples workshop on relational satisfaction and communication patterns. Journal of Couple \& Relationship Therapy, 15, 1-18. doi:10.1080/15332691.2014.978061

Schrodt, P., Witt, P. L., \& Shimkowski, J. R. (2014). A meta-analytical review of the demand/withdraw pattern of interaction and its associations with individual, relational, and communicative outcomes. Communication Monographs, $\quad 81, \quad 28-58$. doi:10.1080/03637751.2013.813632

Stewart, J. W., Bradford, K., Higginbotham, B. J., \& Skogrand, L. (2016). Relationship helpseeking: A review of the efficacy and reach. Marriage \& Family Review, 52(8), 781-803. doi:10.1080/01494929.2016.1157559

Sullaway, M., \& Christensen, A. (1983). Assessment of dysfunctional interaction patterns in couples. Journal of Marriage and the Family, 45, 653-660. doi:10.2307/351670

Vanhee, G., Lemmens, G. M. D., Stas, L., Loeys, T., \& Verhofstadt, L. L. (2018). Why are couples fighting? A need frustration perspective on relationship conflict and dissatisfaction. Journal of Family Therapy, 40, S4-S23. doi:10.1111/1467-6427.12126

Watzlawick, P. (2014). The interactional view. In E. M. Griffin, A. Ledbetter, \& G. Sparks (Ed.), A first look at communication theory (9. ${ }^{a}$ ed., pp.164-174). Nova Iorque: McGrawHill.

Winstok, Z., \& Smadar-Dror, R. (2018). Gender, escalatory tendencies, and verbal aggression in intimate relationship. Journal of Interpersonal Violence. doi:10.1177/0886260518805764 